This is the peer-reviewed version of the article:

Stevanović, V. B.; Pantović, A.; Krga, I. S.; Zeković, M. T.; Šarac, I. R.; Glibetić, M. D.; Vidović, N. Đ. Aronia Juice Consumption Prior to Half-Marathon Race Can Acutely Affect Platelet Activation in Recreational Runners. Applied Physiology Nutrition \& Metabolism 2020, 45 (4), $393-$ 400. https://doi.org/10.1139/apnm-2019-0267.

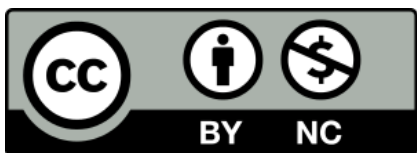

This work is licensed under the Creative Commons Attribution-NonCommercial 4.0 International license 


\title{
ARONIA JUICE CONSUMPTION PRIOR TO HALF-MARATHON RACE CAN ACUTELY AFFECT PLATELET ACTIVATION IN RECREATIONAL RUNNERS
}

\author{
Authors: Stevanović Vuk ${ }^{1 *}$, Pantović Ana ${ }^{*}, K_{\text {rga Irena }}{ }^{1}, Z_{\text {Zeković Milica }}{ }^{1}$, Šarac Ivana ${ }^{1}$, \\ Glibetić Maria ${ }^{1}$, Vidović Nevena ${ }^{1}$. \\ ${ }^{1}$ Institute for medical research, Centre of Research Excellence in Nutrition and Metabolism, \\ University of Belgrade, Tadeuša Košćuškog st. 1, 11000 Belgrade \\ * First authors
}

Coresponding author:

dr Stevanović Vuk

Tadeuša Košćuškog st. 1, 11000, Belgrade

phone: +381642474135; e-mail: vuk.stevanovic@,imi.bg.ac.rs

Other e-mails: kaya 7b@hotmail.com ; 1 renakrga@yahoo.com; zekovicmilica@gmail.com; ivanasarac@yahoo.com; mglibetic@gmail.com; nevenakardum@gmail.com 


\begin{abstract}
Long-distance running, especially in non-professional runners, can increase cardiac arrest risk by enhancing platelet activation and aggregation. Polyphenols can exert cardioprotective effects by positively influencing platelet function. This study aimed to examine the acute effects of polyphenol-rich aronia juice consumption, before simulation of a half-marathon race, on platelet activation and aggregation with leukocytes in recreational runners. In this acute cross-over study, 10 healthy male runners (age $30.8 \pm 2.3$ years) consumed breakfast with $200 \mathrm{ml}$ of aronia juice or $200 \mathrm{ml}$ of placebo. They warmed-up and ran a simulated half-marathon race $(21.1 \mathrm{~km})$. Blood was collected at baseline, $15 \mathrm{~min}, 1 \mathrm{~h}$ and $24 \mathrm{~h}$ after the run. All variables were analysed with 4(Time)x2(Group) analysis of variance with repeated measures on both factors. Results revealed a significant effect of Group on platelet activation parameters: P-selectin and GPIIb-IIIa expressions significantly decreased in aronia compared with the placebo $\operatorname{arm}(\mathrm{F}(1,9)=10.282$, $\mathrm{p}=0.011$ and $\mathrm{F}(1,9)=7.860, \mathrm{p}=0.021$, respectively). The effect of Time was significant on both platelet aggregation markers: platelet-monocyte and platelet-neutrophil aggregates were significantly lower after the race $(F(3,7)=4.227, p=0.014 \operatorname{andF}(3,7)=70.065, p=0.000$, respectively), with changes more pronounced in the later. All effects remained when platelets were exposed to an agonist. These results suggest that aronia consumption could counteract the half-marathon race-induced changes in platelet function.
\end{abstract}

\title{
Key Points
}

- Aronia juice consumption significantly decreased the expression of platelet activation markers, but didn't affect platelet aggregation.

- The race itself did significantly reduce platelet-neutrophil aggregation. 
- Aronia juice may serve as a supplement beverage for recreational runners, to alleviate enhanced platelet reactivity caused by prolonged running.

Key words: platelets, activation, aggregation, aronia, running, half-marathon 


\section{INTRODUCTION}

During the last decade, recreational and long distance running (i.e., half-marathons, marathons and ultramarathons) have gained great popularity, with the number of participants and races increasing year after year (Burkule 2016; Predel 2014). Runners have a lower risk of allcause and cardiovascular disease mortality compared with non-runners while persistent running over time is associated with mortality reduction (Lee et al. 2014). However, long-distance running can pose challenges to runner's cardiovascular system and it has been associated with a higher risk of acute cardiac events, especially in untrained runners, who are less physically fit (Burkule 2016; Predel 2014), compared with professional athletes. Moreover, this risk increases as the length and the number of races rise (Tunstall Pedoe 2007). One of the potential mechanisms by which acute intensive activity (such as half-marathon running) may affect the vascular system and thus enhance the cardiac arrest risk is by altering platelet function and promoting their activation and subsequent aggregation with other blood cells.

Activated platelets are essential for the formation of blood clots to stop bleeding and heal wounds (Baeza et al. 2017). However,excessive platelet activation and aggregation have been associated with thrombosis and adverse cardiovascular events such as stroke and myocardial infarction (Finsterbusch et al. 2018). Studies report that exposure to acute physical activity increases platelet activation and aggregation, however, these results are conflicting (Heber and Volf 2015). The discrepancy in these results might be due to the difference in the type of physical activity, the training status of the participants, but also to the different methodology applied. 
Polyphenols are phytochemicals widely distributed in fruits, vegetables and plant-derived beverages, with a reported positive impact on cardiovascular health. Cardioprotective effects of polyphenols are partly associated with their ability to modulate platelet function. Several dietary intervention studies with polyphenols or polyphenol-rich foods indeed report that these compounds affect disturbed platelet function by reducing platelet activation and aggregation with other platelets and leukocytes.

The effects of consumption of various polyphenol-rich fruit juices were investigated in recreational and elite runners. Tart cherry juice consumption was found to increase total antioxidant capacity, reduce inflammation and aid the recovery of muscle function(Howatso et al. 2010), while consuming montmorency cherry juice reduced the development of upper respiratory tract symptoms in recreational marathon runnners (Dimitriou et al. 2015). In both studies, the participants consumed the juices before and after running a marathon race. Grape juice supplementation managed to improve the total antioxidant capacity and to reduce inflammation in recreational runners after being exposed to time-to-exhaustion exercise test, anaerobic threshold test and aerobic capacity test (Toscano et al. 2015). One of the most promising sources of polyphenols is aronia (Aronia melanocarpa), which diverse beneficial effects have been reported in human intervention trials (Kardum et al. 2015), including the capacity to modulate platelet activation and aggregation as well (Sikora et al. 2012). However, the potential effect of aronia consumption on physical activity-induced modulations in platelet function has not been investigated.

Therefore, the aim of this study was to examine the acute effects of aronia juice consumption before a simulation of half-marathon race on platelet activation and aggregation 
with leukocytes, in amateur recreational runners. We hypothesized that the half-marathon race would increase both platelet activation and aggregation, while the intake of aronia juice polyphenols prior to the race would ameliorate these changes.

\section{METHODS}

\section{Study design}

A single blind cross-over placebo-controlled study was designed to determine acute effects of aronia juice consumption before a simulated half-marathon race on platelet function in healthy recreational long-distance runners. Participants received two different dietary treatments (aronia juice or placebo) and performed half-maraton runs in a counterbalanced order, seven days apart. Each session consisted of a 10-minute rest period, followed by first blood sampling (T0). Participants then had unified breakfast and consumed aronia juice or placebo. After approximately $35 \mathrm{~min}$, which were used for assessment of participants' dietary intake, they warmed-up for approximately $10 \mathrm{~min}$, and run a half-marathon simulation race $(21.1 \mathrm{~km})$. Blood sampling was performed 15min (T1), $1 \mathrm{~h}$ (T2) and 24h (T3) after the run (Figure 1).

Although the plasma concentrations of polyphenols following the consumption of aronia juice was not measured, this study design was based on the results from the available aronia bioavailability studies in humans(Istas et al. 2019; Wiczkowski, Romaszko, \& Piskula 2010; Xie et al. 2016). Anthocyanins reach maximal plasma concentrations at $1 \mathrm{~h}$ to $1.6 \mathrm{~h}$ following the consumption of aronia juice/extract (Wiczkowski et al. 2010; Xie et al. 2016). Furthermore, various phenolic acids, flavanols and their metabolites are detected in circulation by $2 \mathrm{~h}$ following aronia intake (Istas et al. 2019; Xie et al. 2016). Consequently, the timing of aronia consumption was selected, so that peak anthocyanin concentration, as well as substantial levels of total plasma 
polyphenols, would occur in circulation during the exercise, and potentially affect platelet reactivity in response to running.

---- Figure 1 about here -----

\section{Subjects}

Ten apparently healthy recreational male runners, members of Belgrade Urban Running Team, participated in the study (age $30.8 \pm 2.3$ years; $185.3 \pm 7.4 \mathrm{~cm} ; 84.3 \pm 13.5 \mathrm{~kg}$; body mass index (BMI) $24.6 \mathrm{~kg} / \mathrm{m}^{2}$ ). For inclusion in the study, subjects had to meet the following eligibility criteria: a) age between 25 and 35 years, b) body mass index in range of $18-30 \mathrm{~kg} / \mathrm{m}^{2}$, c) non-smokers, d) active members of Belgrade Urban Running Team with four practices per week (approximately $40 \mathrm{~km}$ of weekly running distance), e) no use of medication, f) no relevant medical history, g) no food allergy or intolerance to aronia juice components, h) no blood donations for transfusion purposes within the previous month.

Participants were asked to maintain the same level of physical activity and not to alter their regular dietary habits during the study. They were instructed to rest at least $36 \mathrm{~h}$ prior to half-marathon runs and abstain from the consumption of polyphenol-rich foods such as coffee, tea, cocoa, chocolate, berries, fruit juices and wine $48 \mathrm{~h}$ prior to, and during, the study. Written informed consent was obtained from all the participants. The study was conducted according to the Declaration of Helsinki and the protocol was approved by the Ethics Committee of Ethical Committee of the Faculty of Sport and Physical Education, University of Belgrade, Serbia (ref. no. 02-1072/18-1). All participants were fully informed and prepaired for the procedures used in this research and could withdraw from the study at any time without penalty. 


\section{Blood sampling and analyses}

Venous blood samples were obtained after an overnight fast (T0) and at each time point following the running (T1-T3). Prior to each blood sampling, participants rested for at least $10 \mathrm{~min}$. For the analyses of platelet function, whole blood samples were collected, according to the previous guidelines (Krueger et al. 2002), into vacationer tubes containing sodium citrate. Biochemical parameters were assessed in samples collected into sample tubes for serum using the clinical chemistry analyzer Cobasc111 and reagent kits (Roche Diagnostics, Basel, Switzerland), as recommended by the manufacturer. Complete blood count was determined in samples collected into EDTA evacuated tubes using the automated hematological analyzer ABX Micros 60 (Horiba, Kyoto, Japan).

\section{Flow cytometry/determination of platelet function}

Expression of platelet activation markers (P-selectin and GPIIb-IIIa) and paleteletleukocyte aggregates were analyzed by whole blood flow cytometry according to previously published protocols (Barnard et al. 2003; Krueger et al. 2002). Analyses were performed with the FACS Calibur flow cytometer using the CellQuest software version 6.0. (Becton Dickinson, Franklin Lakes, USA). For the measurements of platelet activation, whole blood aliquots were dissolved (1:10) in Hepes-Tyrode Buffer (HTB) and incubated with peridinin chlorophyll protein (PerCP)-conjugated anti-CD61, fluorescein isothiocyanate (FITC)-conjugated PAC1 (antiGPIIb-IIIa) and phycoerythrine (PE)-conjugated CD62P (anti-P-selectin) monoclonal antibodies with or without a suboptimal concentration $(0.5 \mu \mathrm{M})$ of platelet agonist ADP for $20 \mathrm{~min}$ at room temperature, in the dark. Afterwards, samples were fixed for $15 \mathrm{~min}$ with fixing buffer and 
analyzed. Results were expressed as a percentage of P-selectin and GPIIb-IIIa positive platelets in the total number of collected platelets (20000).

For the platelet-leukocyte (monocyte and neutrophil) aggregates analyses, aliquots of whole blood samples were incubated for $15 \mathrm{~min}$ in the dark, at room temperature with FITCconjugated anti-CD61, PerCP-conjugated anti-CD14, and PE-conjugated anti-CD11b antibodies with or without $0.5 \mu \mathrm{M}$ ADP. Subsequently, samples were treated for $10 \mathrm{~min}$ with erythrocytelysing buffer, washed twice with HTB, fixed for $15 \mathrm{~min}$ in fixing buffer and analyzed. Results were expressed as a percentage of aggregates in the total population of monocytes (1000) and neutrophils (10000).

All chemicals, including monoclonal antibodies, Lysing Solution and Cell Fix Solution were obtained from Becton Dickinson (Franklin Lakes, USA).

\section{Breakfast}

After baseline assessments (T0) and one hour prior to the race, the participants were provided with identical breakfast consisting of three slices of white bread, three slices of chicken breast, $10 \mathrm{~g}$ of butter, and one banana. Two of the participants were following a vegan diet, thus their breakfast comprised of two slices of white bread, $75 \mathrm{~g}$ of hummus and one banana. Both types of breakfast were similar in calories (480.55 and $478.5 \mathrm{kcal}$ for the non-vegan and vegan breakfast, respectively), as well as in their macronutrient composition (the non-vegan breakfast comprised of $80.11 \mathrm{~g}$ of carbohydrates, $13.74 \mathrm{~g}$ of proteins and $11.42 \mathrm{~g}$ of fat, while vegan breakfast contained $80.41 \mathrm{~g}$ of carbohydrates, $11.04 \mathrm{~g}$ of proteins, and $12.36 \mathrm{~g}$ of fat). All of the study participants were breakfast consumers, not breakfast skippers. Shortly after breakfast, participants consumed $200 \mathrm{ml}$ of aronia or placebo juice. 


\section{Aronia juice and placebo}

The polyphenol-rich aronia and polyphenol-free placebo juices were donated from Rheapharm d.o.o., Belgrade, Serbia. Total phenolic content in aronia juice was $1296.8 \mathrm{mg}$ of gallic acid equivalents (GAE) per 200ml. The detailed characterization of phenolic compounds present in juice was previously published(Tomić et al. 2016). The placebo juice had the same nutrient (macro and micronutrient) content with the lack of polyphenols and addition of artificial colors and flavors (Kardum et al. 2017). The formulation of the placebo juice used in this trial was previously developed by our group, with the aim to serve as a control beverage in aronia juice intervention studies. What is more, we concluded that this placebo beverage could be used as a control juice without imposing an effect on platelet function, or other related cardiovascular risk parameters. Both the aronia and placebo juice were evaluated by a team of six food panelists, who confirmed the placebo juice was identical to the aronia juice in terms of the taste, color, smell and texture. The participants tolerated the placebo juice very well, without experiencing any side effects (Kardum et al. 2017).

\section{Dietary intake assessment}

Dietary intake data were obtained via repeated $24 \mathrm{~h}$ dietary recalls within structured personal interviews conducted by trained researchers. These assessments were performed following multiple-pass technique, standardized protocol, probing methods and quality-assurance procedures. Food consumption data were collected for three non-consecutive days - two work days and one weekend day. In order to facilitate reporting, improve recall accuracy and precision 
of quantification, participants were provided with validated Food atlas i.e. booklet comprising photograph-series of reference portions for 135 items i.e. simple foods and composite dishes commonly consumed in the Balkan region (Nikolić et al. 2018).

Dietary data were analyzed using Diet Assess \& Plan, an advanced platform for standardized food consumption data collection and comprehensive nutritional assessment, previously evaluated by European Food Safety Authority (EFSA). Energy, macro- and micronutrient intakes from $24 \mathrm{~h}$ recalls' data were calculated using the mean values of the three administrations, and based on Serbian Food Composition Database, developed in accordance with international standards and hosted on EuroFIR platform(Gurinović et al. 2016). Each participant's recall data was compared against nutritional recommendations for carbohydrates, protein and fat intake specific to demographic characteristics (gender, age, anthropometric measurements) and level of physical activity (Kerksick et al. 2018). Quantitative assessment of micronutrient adequacy was based on Dietary Reference Values (DRVs) proposed by EFSA with Average requirements (AR) and Adequate intake (AI) applied as reference standards (European Food Safety Authority (EFSA) 2017).

\section{Warm up and half-marathon simulation}

All subjects could organize their own warm-up and stretch for 10min before performing a half marathon run $(21.1 \mathrm{~km})$ on a flat, off-road track. They were asked to run the course as fast as possible at their self-selected pace. All subjects run in pairs, in the morning, on the same track and similar weather conditions (temperature: $8-14^{\circ} \mathrm{C}$; humidity $45-55 \%$; wind: $2-4 \mathrm{~m} / \mathrm{s}$ ).

\section{Statistical analyses}


SPSS software, version 20.0 (SPSS, Chicago, USA), was used for the statistical analysis of data. Baseline values were compared between groups by t-test for dependent samples.For assessment of variability between and within groups, all values were normalized relative to their corresponding T0 values andexpressed as percentages of the mean value at $\mathrm{T} 0$, for each participant separately (formula: $(\mathrm{Tx}-\mathrm{T} 0) / \mathrm{T} 0 * 100)$. All variables were analysed using a 4 (Time variable: T0, T1, T2, T3) x 2 (Group variable: aronia juice vs placebo consumption) analysis of variance (ANOVA) with repeated measures on both factors. Post hoc pair-wise analyses following ANOVA were carried out using the Bonferroni correction to determine the significance of both factors, and the interaction of the factors. Statistical results were considered significant if $\mathrm{p}<0.05$.

\section{RESULTS \\ Baseline characteristics and dietary intake of the study participants}

----- Table 1. about here ------

Table 1. represents the baseline characteristics of the study participants. The biochemical and hematological parameters were within the normal reference ranges.

\section{------ Table 2. about here ------}

Dietary intake data is presented in Table 2. Estimated contribution of fat to total energy intake (TEI) exceeded the recommended 20-35\% TEI, whereas carbohydrate intake was slightly 
below acceptable macronutrient distribution range (AMDR) (i.e. 45-65\% TEI). Frequency analyses revealed that $8 / 10$ participants failed to meet recommendations proposed by the International Society of Sports Nutrition for exercising individuals regarding adequate protein intake (1.4-2.0g/kg body weight/day). With regards to micronutrient intake analyses, seven subjects reached AI levels for vitamin E and manganese, while three of them met recommendations for magnesium and copper (Table 2). Seven study participants had adequate intake of zinc (applying recommendations defined for moderate dietary phytate intake), while only four surpassed selenium AI level. Half of the runners had inadequate intake of $767.62 \pm 243.65 \mathrm{mg} /$ day calcium. Based on average requirements cut-point method, vitamin C inadequacy was present in four runners.

\section{Effects of the simulated half-marathon race and aronia juice consumption on platelet}

\section{function}

There were no notable differences in the time needed to complete a half-marathon race between subjects that consumed aronia and placebo drink. In aronia group, runners completed the race by an average $121 \mathrm{~min}$ and in placebo group by $112 \mathrm{~min}$. The time needed to end the race can indirectly give information about the exercise intensity. Thus, these results suggested that both groups ran at a similar intensity level.

Two way repeated measures ANOVA revealed a significant effect of Group

$\left(F_{(1,9)}=10.282, p=0.011\right)$, whereas the effects of Time $\left(F_{(3,7)}=1.698, p=0.191\right)$ and Time $x$ Group interaction $\left(\mathrm{F}_{(3,7)}=1.631, \mathrm{p}=0.205\right)$ were not significant on the expression of P-selectin in the basal state. The participants experienced a 19.65\% lower expression of P-selectin (basal state) when they consumed aronia as opposed to the arm when they consumed placebo juice. (Fig. 2A). 
Similarly, there was a significant effect of Group $\left(\mathrm{F}_{(1,9)}=12.768, \mathrm{p}=0.006\right)$, and a nonsignificant of Time $\left(\mathrm{F}_{(3,7)}=2.867, \mathrm{p}=0.055\right)$ and Time $\mathrm{x}$ Group interaction $\left(\mathrm{F}_{(3,7)}=2.701, \mathrm{p}=0.065\right)$ on the expression of P-selectin in response to the agonist (ADP). The aronia arm showed a $16.85 \%$ decrease in the expression of P-selectin when exposed to ADP compared with the placebo arm (Fig. 2B).

There were significant effects of Group $\left(\mathrm{F}_{(1,9)}=7.860, \mathrm{p}=0.021\right)$ and Time $\left(\mathrm{F}_{(3,7)}=3.021\right.$, $\mathrm{p}=0.047$ ) on the expression of GPIIb-IIIa in the basal state, while the effect of Time $\mathrm{x}$ Group interaction $\left(\mathrm{F}_{(3,7)}=2.912, \mathrm{p}=0.107\right)$ was not significant. As in the case of P-selectin, the expression of GPIIb-IIIa was significantly lower when the participants consumed aronia than placebo juice (42.69\% lower), while the post-hoc tests for the Time effect did not reveal any significant differences between the specific time-points. The analysis of the expression of GPIIbIIIa after stimulation with ADP, showed a significant effect of Group $\left(F_{(1,9)}=9.675, p=0.013\right)$ while the Time effect $\left(\mathrm{F}_{(3,7)}=2.728, \mathrm{p}=0.113\right)$ and Time $\mathrm{x}$ Group interaction $\left(\mathrm{F}_{(3,7)}=2.738\right.$, $\mathrm{p}=0.124)$ were not significant. The post-hoc analysis showed a significant $4.62 \%$ decrease in the expresson of GPIIb-IIIa (after simulation with ADP) when the participants consumed aronia juice than when they consumed placebo (Fig. 2D).

---- Figure 2 about here ----

There was a significant effect of Time $\left(\mathrm{F}_{(3,7)}=4.227, \mathrm{p}=0.014\right)$, but effects of Group $\left(\mathrm{F}_{(1,9)}=1.885, \mathrm{p}=0.203\right)$ and Time $\mathrm{x}$ Group interaction $\left(\mathrm{F}_{(3,7)}=2.645, \mathrm{p}=0.069\right)$ had no significant effects in the proportion of platelet-monocyte aggregates (PMA) in the basal state. The 
percentage of PMAs was significantly lower by $29.97 \%$ h (T2) than $24 \mathrm{~h}$ after the race (T3) (Fig. 3A).

Similar results were obtained after analyzing the proportion of PMA after simulation with ADP. The effect of Time $\left(\mathrm{F}_{(3,7)}=3.625, \mathrm{p}=0.026\right)$ was significant, while effects of the Group $\left(\mathrm{F}_{(1,9)}=0.355, \mathrm{p}=0.566\right)$ and Time $\mathrm{x}$ Group interaction $\left(\mathrm{F}_{(3,7)}=1.375, \mathrm{p}=0.272\right)$ were not. The posthoc analysis confirmed that the percentage of these aggregates significantly increased in T3 compared with T2 by $26.63 \%$ (Fig. 3B).

The analysis further showed that Time imposed a significant effect $(\mathrm{F}(3,7)=70.065$, $\mathrm{p}=0.000$ ), while the effect of Group and Time $\mathrm{x}$ Group interaction a non-significant on the formation of platelet-neutrophil (PNA) aggregates in the basal state $(F(1,9)=0.283, p=0.608$, and $\mathrm{F}(3,7)=0.280, \mathrm{p}=0.839$, for Group and Time $\mathrm{x}$ Group interaction, respectively). Further analysis showed that the proportion of PNAs was significantly lower in T1 and T2 compared with T0 (lower by $37.70 \%$ and $43.66 \%$, respectively), and compared with T3 (lower by $34.40 \%$ and 40.37\%, respectively) (Fig. 3C).

These findings were confirmed when ADP was added as an agonist as well. We observed a significant effect of Time $(\mathrm{F}(3,7)=60.556, \mathrm{p}=0.000)$, while Group and Time $\mathrm{x}$ Group interaction did not reach the significance threshold $(\mathrm{F}(1,9)=0.879, \mathrm{p}=0.373$, and $\mathrm{F}(3,7)=1.077, \mathrm{p}=0.375$, for Group and Time $\mathrm{x}$ Group interaction, respectively). The proportion of PNAs (after simulation with ADP) was significantly lower in T1 and T2 compared with T0 (by $40.51 \%$ and 50.29\%, respectively), and compared with T3 as well (for $32.84 \%$ and $42.61 \%$, respectively). Finally, the proportion of formed PNAs was significantly lower in T1 compared with T2 by $9.78 \%$ (Fig. 3D). 


\section{DISCUSSION}

This study examined the acute effects of aronia juice consumption before a simulation of half-marathon race on markers of platelet activation and aggregation, in recreational runners. We report for the first time that aronia polyphenols were able to ameliorate changes in platelet function induced by running a simulated half-marathon race. Aronia juice consumption prior to the race simulation, significantly decreased the expression of platelet activation markers (Pselectin and GPIIb-IIIa), with or without the addition of ADP as an agonist. It did not affect platelet aggregation, measured as the percentage of formed aggregates between platelets and monocytes, and platelets and neutrophils. However, the race itself significantly affected the levels of these aggregates, with significant changes in the post- compared with the pre-race values observed only in the case of the percentage of PNA. Interestingly, the PNA levels showed a significant decrease following the half-marathon race simulation, and returned to the baseline levels after $24 \mathrm{~h}$. Our results imply that aronia juice may serve as a supplement beverage for recreational runners, in order to prevent enhanced platelet reactivity caused by prolonged running.

Well-balanced, diverse diet with adequate nutrient intake promotes achievement of training goals, supports tissue growth and adaptation, and enables appropriate post-exercise recovery (Thomas et al. 2016). In addition, antioxidative nutrients have received significant attention in the field of sport nutrition over the last few decades, since scientific evidence accumulated linking strenuous physical activity with increased risk of acute cardiovascular events (Burkule 2016). The assessment of self-reported dietary intake in our study revealed that substantial number of examinees had inadequate intake of nutrients with antioxidative properties 
with particular emphasis on copper and vitamin C. Although there is on-going debate on the effectiveness and safety of antioxidant supplementation during training, there is scientific consensus concerning the importance of well-balanced and diverse diet rich in antioxidants for all physically active individuals (Clarkson and Thompson 2000; Thomas et al. 2016). Current recommendations suggest that adequate physiological antioxidant status should be achieved via consumption of proper quantities of antioxidant compounds derived from fruits, vegetables, nuts and whole grains (Thomas et al. 2016). The focus of our research was thus directed towards aronia juice, as good natural source of potent polyphenol antioxidants, with to aim to establish their potential platelet-promoting effect.

Platelets are small non-nucleus cells, which have an essential role in homeostasis, by forming blood clots at the sites of vascular injury. However, these cells are highly sensitive, and if activated (due to oxidative or exercise-induced stress) the development of thrombotic and immunologic adverse events may occur (Finsterbusch et al. 2018).

In our trial, we determined the levels of platelet activation by measuring the expression of P-selectin and GPIIb-IIIa. P-selectin is an adhesion molecule present in platelet $\alpha$-granules. Upon platelet activation it rapidly translocates to the cell surface and mediates platelet aggregation with leukocytes by binding P-selectin glycoprotein ligand-1 on leukocytes (Krga et al. 2018). These heterotypic aggregates formed between platelets and leukocytes initiate the release of inflammatory cytokines, the recruitment of specific adhesion molecules and receptors to the cell surface (Finsterbusch et al. 2018) and promote further platelet activation and aggregation processes. 
Clinical trial data support the notion that exercise, depending on the type and intensity, enhances platelet activity, probably due to the change in the hormonal status caused by training. During the physical activity, the cardiac output and the catecholamine levels rise, and studies suggest that platelets are susceptible to catecholamines, and that these hormones may enhance their reactivity(Anfossi and Trovati 1996; Ikarugi et al. 1999).

However, the results of our study imply that the simulated half-marathon race did not induce any significant changes in platelet activation markers. Our results are in line with the studies that report no change in P-selectin positive platelets(Hilberg et al. 2004; Whittaker et al. 2013), as well with the studies that found no change in the expression of GPIIb-IIIa(Coppola et al. 2005; Wang et al. 2006), after physical activity. However, it should be noted that no previous studies investigated platelet-related parameters in a similar model, and that the existing studies differed in terms of agonists and their concentrations used, as well as in the type of exercise protocols applied. Thus, it was difficult to perform comparative analyses of our findings with other studies and reach clear conclusions.

The results of our trial confirmed the initial hypothesis that aronia polyphenols can downregulate the increase in the platelet activity induced by the race, measured by the expression of P-selectin and GPIIb-IIIa.To the best of our knowledge, this is the first dietary intervention trial that investigated the acute effects of the consumption of polyphenol-rich foods on changes in platelet function induced by physical stress. Data from chronic dietary trials, on the other hand, corroborate that supplementation with polyphenol-rich beverages, prior to performing an exercise protocol, can induce favorable effects on platelet function. Three and 4-week long consumption of polyphenol-rich beverages prior to performing either a marathon race or a test on 
the cycle ergometer, managed to ameliorate the changes in platelet function induced by the exercise (Nickel et al. 2016; Santhakumar et al. 2015).

In this study, we used aronia juice which is particularly rich in phenolic acids, anthocyanins and flavanols (Tomić et al. 2016). Available data show that these compounds can affect platelet activation by lowering their P-selectin and/or GPIIb/IIIa expression in response to ex vivo stimulation with different agonists (Baeza et al. 2017; Krga et al. 2018).

We also investigated the effects of the simulated half-marathon race and aronia juice consumption on platelet aggregation markers, expressed as the percentage of formed PMA and PNA in the total population of monocytes and neutrophils. These platelet-leukocytes conjugates represent sensitive markers for the platelet in vivo aggregation and their increased formation has been observed in acute or stable coronary syndromes (Brambilla et al. 2008). The results of our study suggest that aronia juice polyphenols did not induce any effect on platelet aggregation rate with monocytes and neutrophils. However, the simulated half-marathon race managed to significantly decrease the formation of PNA (both with and without the addition of ADP), specifically $15 \mathrm{~min}$ and $1 \mathrm{~h}$ after the race. The significant effect of time in the case of PMA levels was not noteworthy since the post hoc tests revealed no significant difference in the post-race compared with the pre-race values (but only within the post-race values). Our results are not in agreement with most of the studies that assessed exercise-induced changes in the formation of PNA and PMA. Trials report that exercise increases the level of PNA (Li et al. 1999) and PMA (Hilberg et al. 2008; Li et al. 1999), both in the basal and agonist-induced state. Only one study reports that exercise lowered the rate of formed aggregates between monocytes and platelets (Shantsila et al. 2012).Discrepancies between our and results from these studies may be a result 
of the differences in the cardiorespiratory status of the subjects, intensity of physical activity, methodology and type and concentrations of platelet agonist that were used.

However, warm-up may be one important factor which might have led to the decrease in the formation of PNA after the race. Wang et al. (2006) found that high intensity training significantly enhanced the PNA levels, however, when they introduced a 10min warm-up prior to the training, the levels of these aggregates were significantly lower. Our participants performed a warm-up before the race, thus we speculate that it might have lowered the rate of aggregation between platelets and neutrophils.

The same authors further suggest that the observed decrease in platelet aggregation with neutrophils might be due to the dual activity of P-selectin(Wang et al. 2006). When bound to the membrane, P-selectin enhances the aggregation between platelets and leukocytes, however, when present in the fluid phase, it inhibits this interaction (Wang et al. 2006). Although we have not measured soluble P-selectin levels, available trials data report that marathon and o $164 \mathrm{~km}$ road cycling event, induced an increase in soluble P-selectin levels( Kupchak et al. 2016; Zaleski et al. 2013), thus we propose that this was a physiological pattern that occurred in our study as well. Therefore, it is possible that enhanced levels of soluble P-selectin imposed a negative feedback loop on the interaction between platelets and neutrophils induced by the running. However, the exact underlying mechanisms need further investigation.

One of the main strengths of our trial is the cross-over design that allowed us to minimize the potential effects of confounding covariates on our results. In addition, we used flow cytometry, which is considered to be an invaluable technique for assessing platelet function, because it is quick and highly sensitive, and allows platelets to be directly analyzed in their 
native environment, thus minimizing sample manipulation and preventing the potential artificial in vitro activation and loss of platelet subpopulations (Kestin et al., 1993). Other techniques used for the investigations of platelet function face problems of potential artificial in vitro platelet reactivity caused by sample manipulation. In addition, the results of our trial contribute to the current knowledge by providing evidence for implementing a natural beverage as a potent source of polyphenols in the diet. This beverage can help trained men involved in regular physical activity in alleviating the effects when exposed to prolonged exercise, and also meet their requirements for dietary antioxidants. This is in line with the established sports nutrition guidelines which strongly suggest implementing antioxidants in the diet via consumption of whole food and not via supplements, due to the emerging data that warns against consumption of antioxidant supplements in both sports and general population (Bjelakovic et al. 2014; Dutra et al., 2018), in favour of whole foods (Nieman et al. 2018). There are also factors that could have influenced the interpretation of our results. We do not have an insight into the possible interaction of the nutritional components of the breakfast with those of the aronia juice, which might have consequently influenced platelet function. We do not have the information about the previous polyphenol intake of the participants. However, we counteracted this limitation by instructing the participants to refrain from consuming polyphenol-rich foods prior to the study, and by determining their prior consumption of other antioxidant micronutrients. The intake of the major antioxidants was not adequate, thus we propose that there were not any antioxidant compounds present in excess that could have influenced the interpretation of our results. Finally, due to financial constraints, we were not able to perform VO2 max measurements, which is an important determinant of the change in platelet activity after performing an acute exercise (Heber and Volf 2015). It is observed that those with lower cardiorespiratory fitness and lower physical 
activity level experience greater platelet activation than the better-trained counterparts. However, we counteracted this limitation by recruiting healthy men, from the same running team, who had been following the same training regime for a sufficient period of time prior to the study.

\section{CONCLUSIONS}

We report that the consumption of aronia juice just before a half-marathon race simulation was able to impose a positive impact on platelet reactivity in response to running, by lowering the expression of platelet activation markers P-selectin and GPIIb-IIIa in recreational runners. The observed effect might be mediated by phenolic acids, anthocyanins and flavonols present in aronia juice, which anti-thrombotic properties have been previously reported.

Acknowledgments:

This work was supported by the grants (project number III41030) from the Ministry for Education, Science and Technological Development of Republic of Serbia.

Conflict of Interest Statement:

The authors have no conflicts of interests to declare. 


\section{REFERENCES}

Anfossi, G., \& Trovati, M. 1996. Role of catecholamines in platelet function: pathophysiological and clinical significance. Eur. J. Clin. Invest. 26(5), 353-370. doi:10.1046/j.13652362.1996.150293.x PMID: 8796362

Baeza, G., Bachmair, E. M., Wood, S., Mateos, R., Bravo, L., \& De Roos, B. 2017. The colonic metabolites dihydrocaffeic acid and dihydroferulic acid are more effective inhibitors of in vitro platelet activation than their phenolic precursors. Food and Funct. 8(3), 1333-1342. doi: 10.1039/c6fo01404f. PMID: 28229135.

Bjelakovic, G., Nikolova, D., \& Gluud, C. 2014. Antioxidant supplements and mortality. Curr. Opin. Clin. Nutr. Metab. Care. 17(1), 40-44. doi: 10.1097/MCO.0000000000000009. PMID: 24241129.

Brambilla, M., Camera, M., Colnago, D., Marenzi, G., De Metrio, M., Giesen, P.L., Balduini, A. et al. 2008. Tissue factor in patients with acute coronary syndromes. Arterioscler. Thromb. Vasc. Biol. 28(5), 947-953. doi: 10.1161/ATVBAHA.107.161471. PMID: 18292391.

Burkule, N. 2016. Marathon running for amateurs: Benefits and risks. J. Clin. Prev. Cardiol. 5, 113-24. doi: 10.4103/2250-3528.192681

Clarkson, P. M., \& Thompson, H. S. 2000. Antioxidants: what role do they play in physical activity and health? Am. J. Clin. Nutr. 72(2), 637S-646S. doi: 10.1093/ajen/72.2.637S. PMID: 10919970. 
Coppola, A., Coppola, L., dalla Mora, L., Limongelli, F.M., Grassia, A., Mastrolorenzo, L. et al. 2005. Vigorous exercise acutely changes platelet and B-lymphocyte CD39 expression. J. Appl. Physiol. 98(4), 1414-1419. doi:10.1152/japplphysiol.00315.2004. PMID:15772061.

Dutra, M. T., Alex, S., Mota, M. R., Sales, N. B., Brown, L. E., \& Bottaro, M. 2018. Effect of strength training combined with antioxidant supplementation on muscular performance. Appl. Physiol. Nutr. Metab. 43(8), 775-781. doi: 10.1139/apnm-2017-0866. PMID: 29939770.

European Food Safety Authority (EFSA). (2017). Dietary reference values for nutrients summary report. EFSA Supporting Publications, 14(12). doi: 10.2903/sp.efsa.2017.e15121

Finsterbusch, M., Schrottmaier, W. C., Kral-Pointner, J. B., Salzmann, M., \& Assinger, A. 2018. Measuring and interpreting platelet-leukocyte aggregates. Platelets, 29(7), 677-685. doi: 10.1080/09537104.2018.1430358. PMCID: PMC6178087.

Gurinović, M., Milešević, J., Kadvan, A., Djekić-Ivanković, M., Debeljak-Martačić, J., Takić et al. 2016. Establishment and advances in the online Serbian food and recipe data base harmonized with EuroFIR standards. Food Chem, 193, 30-38. doi: 10.1016/j.foodchem.2015.01.107. PMID: 26433284.

Heber, S., \& Volf, I. 2015. Effects of physical (in)activity on platelet function. Biomed Res Int. 1-11. doi: 10.1155/2015/165078. PMID: 26557653.

Hilberg, T., Eichler, E., Gläser, D., Schmidt, V., \& Gabriel, H. H. W. 2004. Platelet activity, reactivity and platelet-leukocyte conjugate formation before and after exhaustive or moderate exercise in patients with IDDM. Platelets, 15(2), 101-108. doi:10.1080/09537100310001646941. PMID: 15154602. 
Hilberg, T., Menzel, K., Gläser, D., Zimmermann, S., \& Gabriel, H. H. W. 2008. Exercise intensity: Platelet function and platelet-leukocyte conjugate formation in untrained subjects. Thromb. Res. 122(1), 77-84. doi:10.1016/j.thromres.2007.08.018. PMID: 17945332.

Ikarugi, H., Taka, T., Nakajima, S., Noguchi, T., Watanabe, S., Sasaki, Y. et al. 1999. Norepinephrine, but not epinephrine, enhances platelet reactivity and coagulation after exercise in humans. J. Appl. Physiol. 86(1), 133-138. doi:10.1152/jappl.1999.86.1.133. PMID: 9887123. Istas, G., Wood, E., Le Sayec, M., Rawlings, C., Yoon, J., Dandavate, V. et al. 2019. Effects of aronia berry (poly)phenols on vascular function and gut microbiota: a double-blind randomized controlled trial in adult men. Am. J. Clin. Nutr. 110(2), 316-329. doi: 10.1093/ajcn/nqz075. PMID: 31152545.

Kardum, N., Konic Ristic, A., Zec, M., Kojadinovic, M., Petrovic-Oggiano, G., Zekovic, M. et al. 2017. Design, formulation and sensory evaluation of a polyphenol-rich food placebo: an example of aronia juice for food intervention studies. Int. J. Food Sci. Nutr. 68(6), 742-749. doi: 10.1080/09637486.2017.1283682. PMID: 28147889.

Kardum, N., Milovanović, B., Šavikin, K., Zdunić, G., Mutavdžin, S., Gligorijević, T., \& Spasić, S. 2015. Beneficial effects of polyphenol-rich chokeberry juice consumption on blood pressure level and lipid status in hypertensive subjects. J. Med. Food. 18(11), 1231-1238. doi: 10.1089/jmf.2014.0171. PMID: 25973889.

Kerksick, C. M., Wilborn, C. D., Roberts, M. D., Smith-Ryan, A., Kleiner, S. M., Jäger, R. et al. 2018. ISSN exercise \& sports nutrition review update: research \& recommendations. J. Int. Soc. Sports Nutr. 15(1), 38. doi:10.1186/s12970-018-0242-y 
Kestin, A. S., Ellis, P. A., Barnard, M. R., Errichetti, A., Rosner, B. A., \& Michelson, A. D. 1993. Effect of strenuous exercise on platelet activation state and reactivity. Circulation, 88(4 I), 1502-1511. doi:10.1161/01.CIR.88.4.1502. PMID: 8403298.

Krga, I., Vidovic, N., Milenkovic, D., Konic-Ristic, A., Stojanovic, F., Morand, C., \& Glibetic, M. (2018). Effects of anthocyanins and their gut metabolites on adenosine diphosphate-induced platelet activation and their aggregation with monocytes and neutrophils. Arch. Biochem. Biophys. 645, 34-41. doi: 10.1016/j.abb.2018.03.016. PMID: 29555206.

Krueger, L. A., Barnard, M. R., Frelinger, A. L., Furman, M. I., \& Michelson, A. D. 2002. Immunophenotypic analysis of platelets. Curr Protoc Cytom. Chapter 6:Unit 6.10. doi: 10.1002/0471142956.cy0610s19. PMID: 18770767.

Kupchak, B.R., Kazman, J.B., Umeda, E.A., Vingren, J.L., Lee, E.C., Armstrong, L.E., \& Deuster, P.A. 2016. Changes in endothelial markers during a summer ultra-endurance road cycling event in the heat. Int. J. Sports Exerc. Med. 2(3). doi: 10.23937/2469-5718/1510045

Lee, D., Pate, R. R., Lavie, C. J., Sui, X., Church, T. S., \& Blair, S. N. 2014. Leisure-time running reduces all-cause and cardiovascular mortality risk. J. Am. Coll. Cardiol. 64(5), 472481. doi: 10.1016/j.jacc.2014.04.058. PMID: 25082581.

Li, N., Wallen, N. H., \& Hjemdahl, P. 1999. Evidence for prothrombotic effects of exercise and limited protection by aspirin. Circulation, 100(13), 1374-1379.

doi:10.1161/01.CIR.100.13.1374. PMID: 10500036.

Michelson, A. D., Barnard, M. R., Krueger, L. A., Valeri, C. R., \& Furman, M. I. 2001. Circulating monocyte-platelet aggregates are a more sensitive marker of in vivo platelet 
activation than platelet surface P-selectin: Studies in baboons, human coronary intervention, and human acute myocardial infarction. Circulation, 104(13), 1533-1537. doi:10.1161/hc3801.095588. PMID: 11571248.

Nickel, T., Lackermair, K., Scherr, J., Calatzis, A., Vogeser, M., Hanssen, H. et al. 2016. Influence of high polyphenol beverage on stress-induced platelet activation. J. Nutr. Health Aging. 20(6), 586-593. doi: 10.1007/s12603-016-0697-y. PMID: 27273347.

Nieman, D. C., Gillitt, N. D., Sha, W., Esposito, D., \& Ramamoorthy, S. 2018. Metabolic recovery from heavy exertion following banana compared to sugar beverage or water only ingestion: A randomized, crossover trial. PLoS ONE 22;13(3):e0194843. doi: 10.1371/journal.pone.0194843. PMID: 29566095.

Nikolić, M., Milešević, J., Zeković, M., Gurinović, M., \& Glibetić, M. 2018. The development and validation of food atlas for portion size estimation in the Balkan region. Front. Nutr. 5, 78. doi: 10.3389/fnut.2018.00078. PMID: 30271776.

Predel, H. G. 2014. Marathon run: Cardiovascular adaptation and cardiovascular risk. Eur. Heart J. 35(44), 3091-3096. doi: 10.1093/eurheartj/eht502. PMID: 24408890.

Santhakumar, A. B., Kundur, A. R., Sabapathy, S., Stanley, R., \& Singh, I. 2015. The potential of anthocyanin-rich Queen Garnet plum juice supplementation in alleviating thrombotic risk under induced oxidative stress conditions. J. Funct. Foods, 14, 747-757. doi:10.1016/j.jff.2015.03.003 
1

2

3

4

5

6

7

8

9

10

11

12

13

14

15

16

17

18

19

20

Table 1. - Baseline biochemical and hematological parameters of the study participants (

\begin{tabular}{cc}
\hline Variable & mean $\pm \mathrm{SD}$ \\
\hline Glucose $(\mathrm{mmol} / \mathrm{L})$ & $4.77 \pm 0.36$ \\
Triglyceridess $(\mathrm{mmol} / \mathrm{L})$ & $0.874 \pm 0.46$ \\
Total Cholesterol $(\mathrm{mmol} / \mathrm{L})$ & $4.55 \pm 0.69$ \\
HDL-cholesterol $(\mathrm{mmol} / \mathrm{L})$ & $1.32 \pm 0.16$ \\
LDL-cholesterol $(\mathrm{mmol} / \mathrm{L})$ & $2.56 \pm 0.58$ \\
Creatinine $(\mu \mathrm{mol} / \mathrm{L})$ & $83.22 \pm 12.30$ \\
LDH & $156.38 \pm 34.47$ \\
PLT $(\mathrm{x} \mathrm{10} / 1)$ & $203.8 \pm 43.74$ \\
PCT (x 10 $\left.{ }^{-2} / / 1\right)$ & $0.17 \pm 0.03$ \\
MPV(fl) & $8.23 \pm 0.87$ \\
PDW & $14.95 \pm 1.53$
\end{tabular}

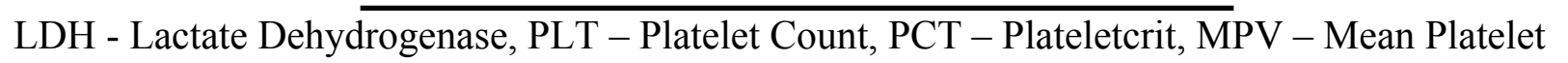
Volume, PDW - Platelet Distribution Width

5
17
18
10


1 Table 2. - Mean daily intake of energy, macronutrients and micronutrients with antioxidative properties.

\section{Nutrients}

\begin{tabular}{|c|c|c|c|c|c|c|c|c|c|c|c|}
\hline $\begin{array}{l}\text { Energy } \\
\text { (kcal) }\end{array}$ & $\begin{array}{c}\text { Carbohydrates } \\
\text { (g) }\end{array}$ & $\begin{array}{l}\text { Protein } \\
(\mathrm{g})\end{array}$ & $\begin{array}{l}\text { Fat } \\
(\mathrm{g})\end{array}$ & $\begin{array}{l}\text { Selenium } \\
(\mu \mathrm{g})\end{array}$ & $\begin{array}{l}\text { Zinc } \\
(\mathrm{mg})\end{array}$ & $\begin{array}{l}\text { Manganese } \\
\text { (mg) }\end{array}$ & $\begin{array}{l}\text { Iron } \\
(\mathrm{mg})\end{array}$ & $\begin{array}{l}\text { Copper } \\
\text { (mg) }\end{array}$ & $\begin{array}{l}\text { Vitamin C } \\
(\mathrm{mg})\end{array}$ & $\begin{array}{l}\text { Vitamin E } \\
(\mathrm{mg})\end{array}$ & $\begin{array}{c}\beta \text {-carotene } \\
(\mu \mathrm{g})\end{array}$ \\
\hline $2302.34 \pm 441.35$ & $248.85 \pm 63.50$ & $89.71 \pm 26.23$ & $105.35 \pm 36.23$ & $61.07 \pm 29.23$ & $10.48 \pm 2.99$ & $3.80 \pm 1.47$ & $11.89 \pm 2.24$ & $1.40 \pm 0.35$ & $121.30 \pm 55.18$ & $19.12 \pm 9.82$ & $4789.15 \pm 2925.02$ \\
\hline
\end{tabular}


Page 30 of 35

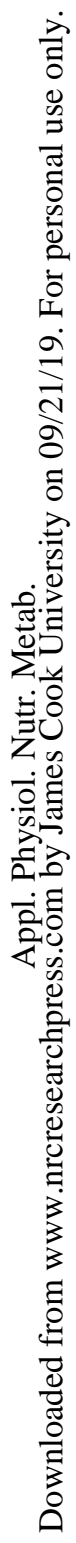




\section{Figure 1}

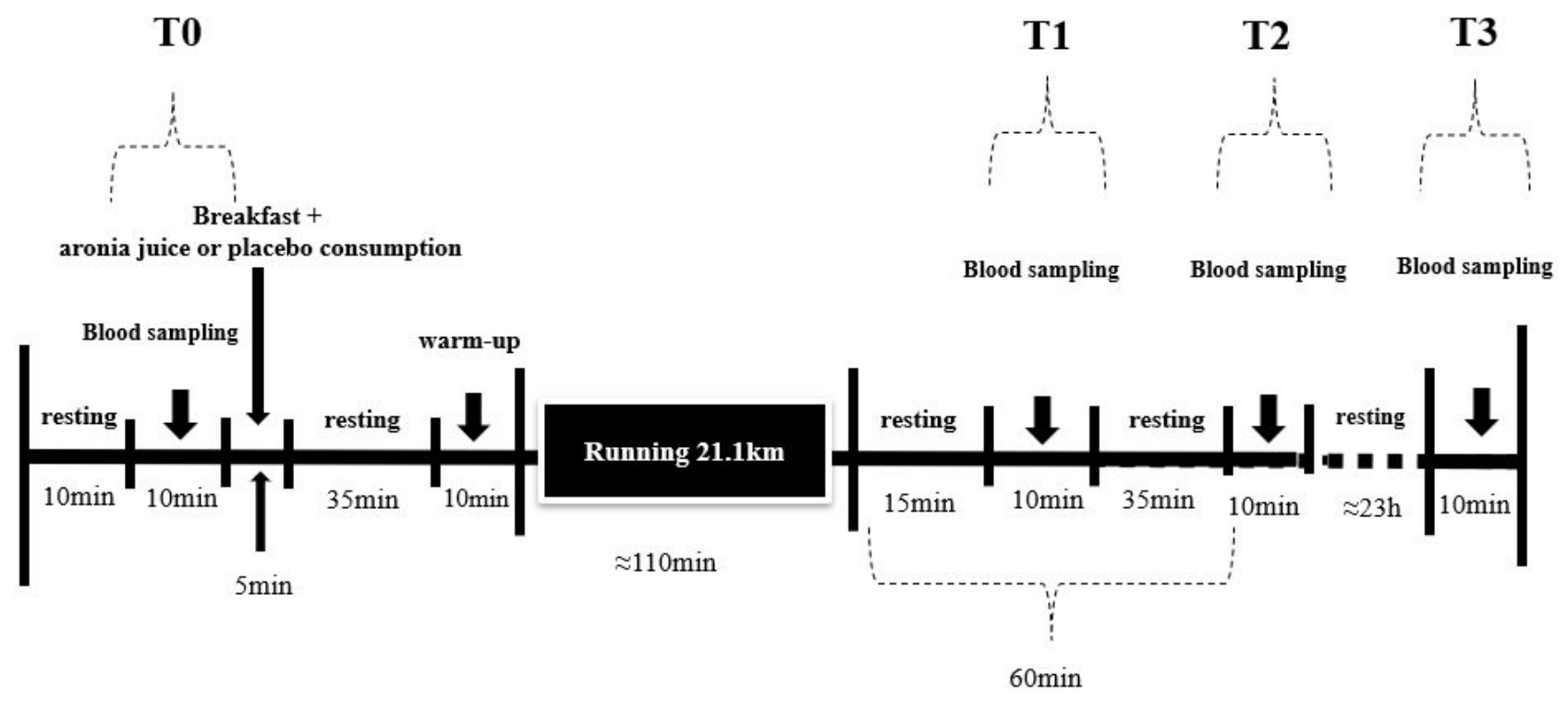

Figure 1. Summary of research design 


\section{Figure 2}
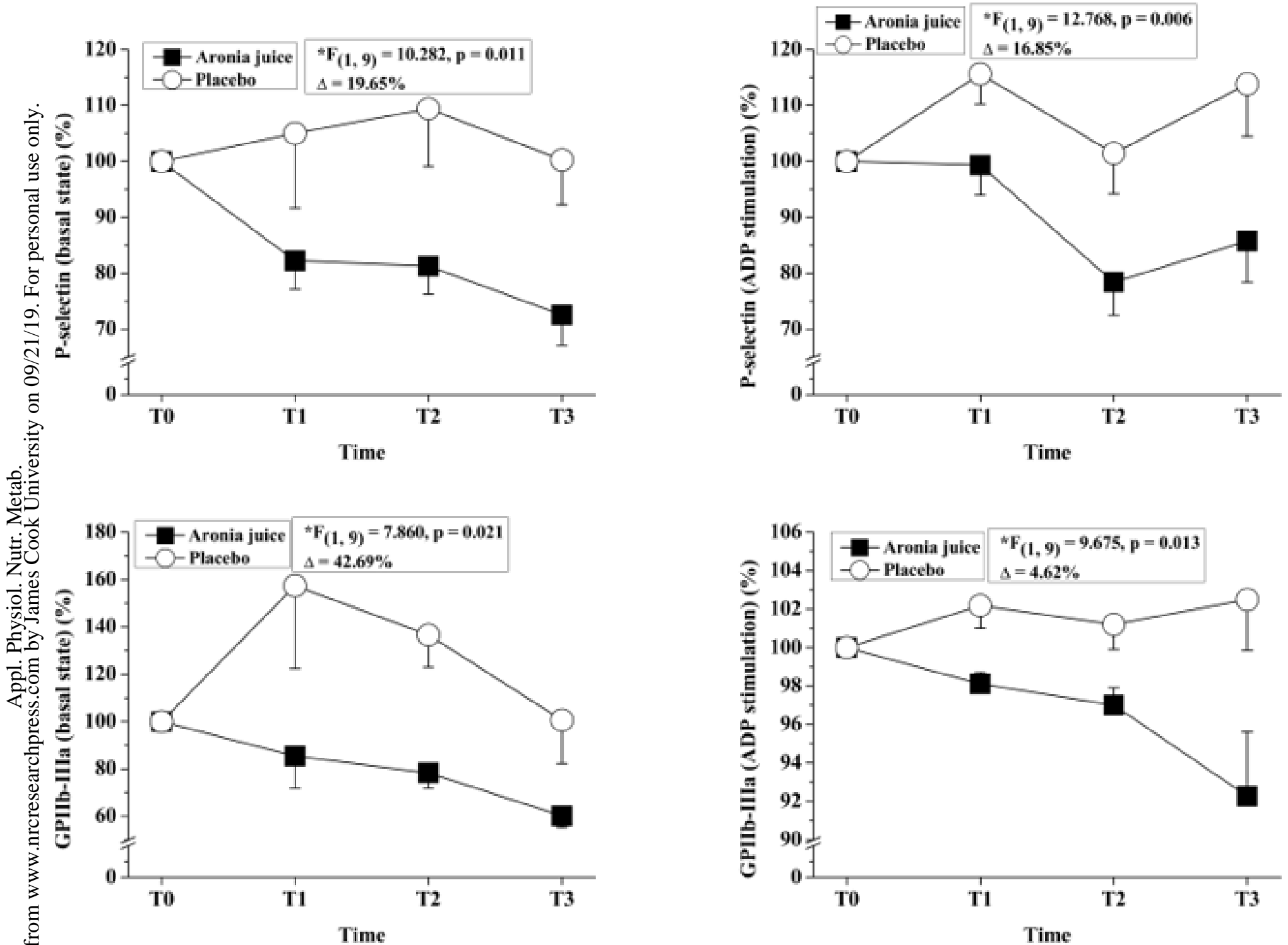

Figure 2. Platelet activation: Comparison of change in the percentage of (A) P-selectin positive platelets (basal state), (B) P-selectin positive platelets (in response to ADP stimulation), (C) GPIIb-IIIa positive platelets (basal state) and (D) GPIIb-IIIa positive platelets (in response to ADP stimulation) after the consumption of aronia and placebo juice at investigated time points. The graph represents results of two-way reapeated measures ANOVA on normalized data. 
Asterics $(*)$ represent a significant effect of Group $(\mathrm{p}<0.05)$, followed by the corresponding differences observed between two arms; 


\section{Figure 3}
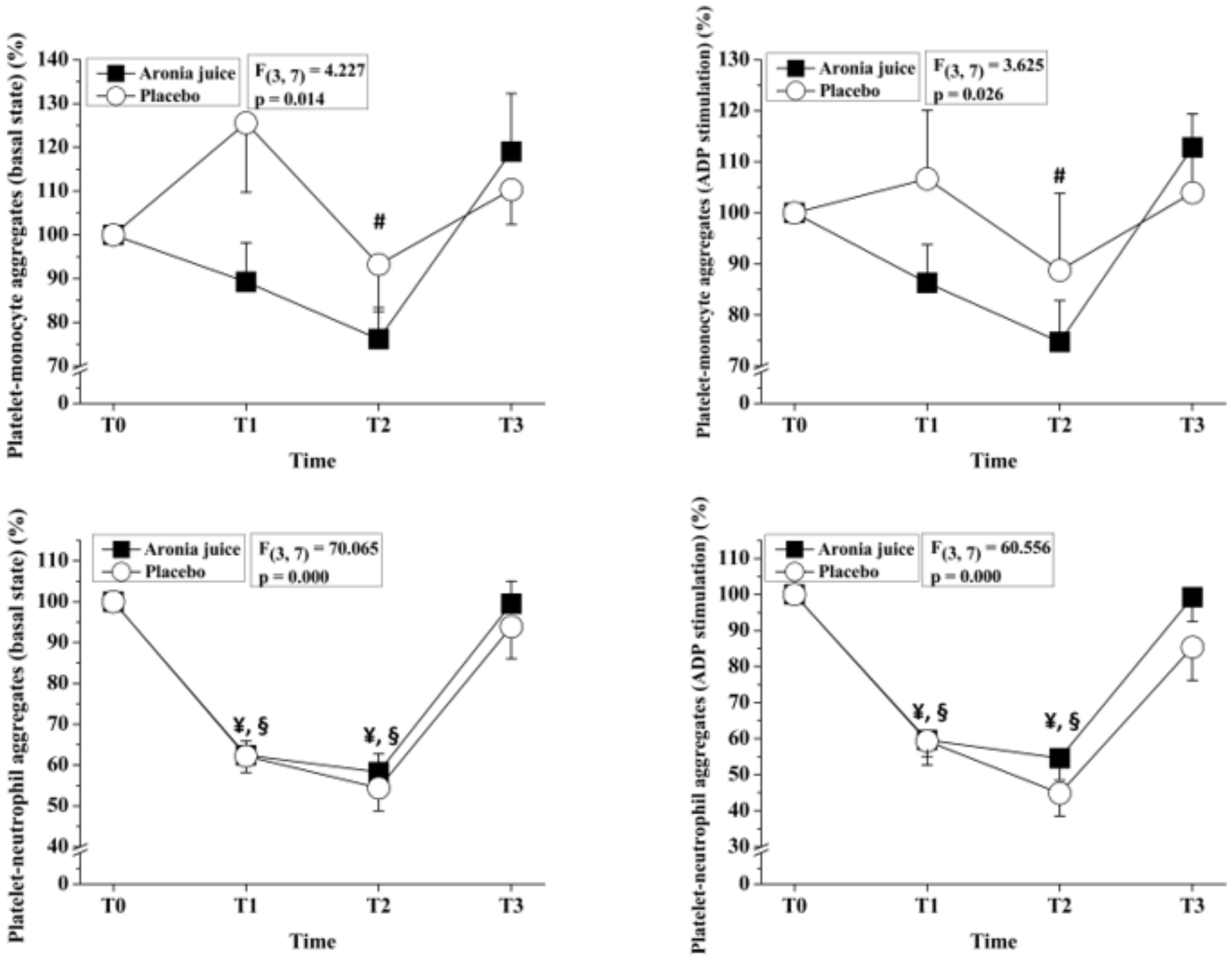

Figure 3. Platelet aggregation: Comparison of change in the percentage of (A) platelet-monocyte aggregates (basal state), (B) platelet-monocyte aggregates (in response to ADP stimulation), (C) platelet-neutrophil aggregates (basal state) and (D) platelet-neutrophil aggregates (in response to ADP stimulation) after the consumption of aronia and placebo juice at investigated time points. The graph represents results of two-way reapeated measures ANOVA on normalized data. 
Hashtag (\#) represents significant effect of Time, specifically denoting differences observed between $\mathrm{T} 2$ and $\mathrm{T} 3$; $¥$ represents significant effect of Time, specifically denoting differences observed in T1 and T2 compared with T0; $§$ represents significant effect of Time, specifically denoting differences observed in $\mathrm{T} 1$ and $\mathrm{T} 2$ compared with $\mathrm{T} 3$. 